\title{
Ultrasound Evaluation of Palpable Breast Masses in Correlation with Fine Needle Aspiration Cytology
}

\author{
Krithika $S^{1}$, Gurubharath llangovan ${ }^{2}$, Harshavardhan Balganesan ${ }^{3}$, Pavithra $A^{4}$ \\ ${ }^{1}$ Junior Resident, Department of Radiodiagnosis, ${ }^{2}$ Professor and HOD, Department of Radiodiagnosis, ${ }^{3}$ Senior Resident, \\ Department of Radiodiagnosis, ${ }^{4}$ Senior Resident, Department of Radiodiagnosis, Shri Sathya Sai Medical College and \\ Research Institute, Chennai, Tamilnadu- 603108, India
}

Corresponding author: Gurubharath Ilangovan, 100 A/5, 11th Sector, 66th Street, PonnambalamSalai KK Nagar, Chennai, India

DOI: http://dx.doi.org/10.21276/ijcmsr.2020.5.2.7

How to cite this article: Krithika S, Gurubharath Ilangovan, Harshavardhan Balganesan, Pavithra A. Ultrasound evaluation of palpable breast masses in correlation with fine needle aspiration cytology. International Journal of Contemporary Medicine Surgery and Radiology. 2020;5(2):B27-B33.

\section{A B S T R A C T}

Introduction: Breast cancer is found to be the leading cancer in woman across the world. Ultrasound is safe and does not have radiation exposure. It is suitable for evaluating young breasts, pregnant, lactating woman. The objectives of our study were to find the accuracy of ultrasound in diagnosis of benign and malignant breast masses and to correlate the benign and malignant ultrasound features with tissue diagnosis.

Materials and methods: This study was a descriptive cross-sectional study which includes 60 female patients, of age ranging between 20-85 years with history of palpable breast masses referred to the Radiology department for ultrasound scan in the past 18 months from January 2018 to June 2019. Initially USG was performed and then FNAC was done. Tissue diagnosis was done in all patients. The sonological findings and FNAC results were correlated with tissue diagnosis by statistical analysis.

Results: The USG findings most predictive for a benign and malignant tissue diagnosis were identified. The sensitivity, specificity, PPV, NPV of ultrasound is $100 \%, 94.3 \%, 89.5 \%, 100 \%$ respectively. False positive and false negative rate of USG is $5.7 \%$ and $0 \%$ respectively. The specificity, sensitivity, NPV, PPV of FNAC is $100 \%, 85 \%, 92.9 \%, 100 \%$ respectively. False negative and false positive rate of FNAC is $7.1 \%$ and $0 \%$ respectively.

Conclusion: Ultrasound and FNAC are supplementary to each other, thus when they are used together, majority of the lesions can be diagnosed. This helps in avoiding radiation exposure \& decrease the number of biopsies performed for benign solid masses.

Keywords: Ultrasound, FNAC, Tissue Diagnosis, Mammography

\section{INTRODUCTION}

Breast cancer is found to be the leading cancer in woman in both developed and developing countries across the world. Cancer incidence in developing countries has been increased due to lack of awareness and detection in late stages. Detection of cancer in the early stage can improve the survival of patients and cancer control can be achieved. ${ }^{1}$ It is estimated that worldwide over 508000 women died in 2011 due to breast cancer ("Global Health Estimates, WHO 2013"). Though breast cancer is thought to be a disease of developed countries, the $50 \%$ of cases and $58 \%$ of deaths occur in developing countries. According to 2014 statistics of WHO the rate of mortality of breast cancer in India was estimated to be $21.5 \%$. Age standardised cancer mortality trends was found highest for breast cancer when compared to all other cancers in India. Breast cancers usually presents as a lump or mass in the breast. It is a major concern to the patient and it need to be evaluated as early as possible. ${ }^{1,2}$

Breast Ultrasonography has evolved as an important complementary modality to mammography, and it is performed along with the mammography for diagnosis of lesions. Sono-mammography can differentiate solid and cystic masses. ${ }^{3,4}$ The image quality has improved the confidence in correct identification of benign lesions and malignant lesions. ${ }^{5,6}$

Although many studies had been conducted in the past, still breast cancer is poorly understood. There are various radiological methods for evaluation of breast. Most commonly employed methods are x-ray-mammogram, sono-mammogram. Ultrasound has more advantages over mammography in diagnosing breast cancer. It has no radiation exposure so it is suitable for evaluating young breasts, pregnant, lactating woman. It helps in diagnosing lesions in dense breasts. The high frequency probes help in providing good lateral resolution. Real time imaging and panoramic views helps in better identification and delineation of the lesion. ${ }^{7,8}$

USG elastography has been evolved recently and the results 
are quite reliable. ${ }^{9}$ Ultrasound is helpful in evaluation of dense breast, even small cancers which were missed in mammography can be picked by ultrasound. ${ }^{10}$

Recently "American College of Radiology" proposed a lexicon for characterization of breast masses. It is an accepted and assured system for characterising the lesions by SonoMammogram, mammogram and MRI. ${ }^{11}$ It is important to recognise the ultrasound features which helps in characterization of breast lesions as it helps in early diagnosis of malignancy and by this unnecessary radiation exposure and invasive procedures can be avoided. ${ }^{12,13}$ BIRADS by ACR helps in practising a standardised reporting system all around the world in characterization of breast masses by Sono-mammogram and conventional mammogram. ${ }^{14}$

The objectives of our study were to find the accuracy of ultrasound in diagnosis of benign and malignant breast masses and to correlate the benign and malignant ultrasound features with tissue diagnosis.

\section{MATERIAL AND METHODS}

This study was a descriptive cross-sectional study conducted in Shri Sathya Sai Medical College and Research Institute, which included 60 female patients, of age ranging between 20-85 years with history of palpable breast masses referred to the Department of Radio- Diagnosis, for ultrasound scan in the past 18 months. Initially USG was performed and then FNAC was done. Tissue diagnosis results were obtained in all patients. The sonological findings and FNAC results were correlated with tissue diagnosis by statistical analysis. Institutional ethical committee clearance was obtained before the initiation of study. Informed written consent was obtained from each patient.

\section{Inclusion Criteria:}

All female patients within 20 to 85 years with palpable breast masses, who underwent FNAC and subsequent biopsy are included.

\section{Exclusion Criteria:}

Patients with breast implants

- Benign breast cysts.

- Male patients

- Woman who underwent mastectomy

\section{Methodology/Procedure}

- Patients with breast lump were referred to our department for ultrasound breast. Initially diagnostic ultrasound was done with Mindray machine using high frequency probe of frequency 7to10 MHz, and then USG guided "Fine needle aspiration cytology" was done in all breast masses and sent for cytopathological examination.

- Following FNAC, depending upon the advice of surgeon, core needle biopsy or excision biopsy was carried out and the tissue diagnosis results were collected.

- Categorization of breast masses is done using Ultrasound Lexicon which was published by American college of Radiology, and previous studies in the literature.

- The Ultrasound findings were then correlated with "Fine needle aspiration cytology" findings. The Ultrasound and FNAC findings are correlated with histopathological findings, considering histopathology as the gold standard.

\section{Patients scanning technique}

Before the ultrasound examination a detailed clinical history and clinical examination was performed. Patients were examined in supine position.

\section{Observations with USG}

A thorough ultrasound examination was performed in sagittal plane, transverse plane and radial plane.

The following observations were made:

Breast composition: [Heterogenous/ Homogenous- Fat / Homogenous- Fibro Glandular]

Quadrant: [Upper Outer (U.O) / Upper Inner (U.I) / Lower Outer (L.O)/ Lower Inner (L.I) / Retro -Areolar (R.A)]

Mass

1. Shape: [Oval / Round / Irregular / >3 Smooth Macro lobulated / <3 Smooth Macro lobulated]

2. Margin: [Circumscribed / Non-Circumscribed / Indistinct / Angular / Micro lobulated / Spiculated]

3. Orientation: [Parallel / Not Parallel]

4. Width/ap ratio: [W/AP RATIO > 1.4/ W/AP RATIO $<1.4]$

5. Echopattern: [Anechoic/ Hyperechoic/ Cystic and solid/ Hypoechoic/ Isoechoic/ Heterogenous]

6. Posterior features: [Unaffected/ Enhancement/ Shadowing/ Mixed]

7. PSEUDOCAPSULE

8. Edge refraction

9. Calcification

Finally, the breast lesions were classified according to BIRADS provided by the "American College of Radiology" to indicate the probability of malignancy. And then each mass is further classified into 3 main categories, Benign (Negative, benign, probably benign), Indeterminate (lesions showing a suspicious abnormality which could not be clearly delineated as benign or malignant), or malignant (Highly suggestive of malignancy).

\section{Observations with FNAC \\ Technique of USG guided FNAC}

After explaining the procedure, under strict aseptic precautions the high frequency linear probe was placed over the mass and under sonographic guidance, the needle was inserted into the lesion after informing the patient about it. After the insertion, rapid back and firm strokes of the needle were made within the lesion. Aspiration was applied using $20 \mathrm{cc}$ syringe attached to the, a 23-gauge needle, for creating negative pressure and before withdrawing the needle, the negative pressure was released. The material in the needle was expressed on clean slides and smeared using another clean slide. Slides were taken to the pathology laboratory and after obtaining the reports from pathologist, the results were grouped into 3 categories (Benign, malignant, indeterminate).

\section{STATISTICAL ANALYSIS}

Data entered in excel sheet, statistical analysis done by SPSS 23 software using descriptive statistics and chi-square test for correlating ultrasound findings with FNAC and tissue 
diagnosis (histopathological examination) in case of breast masses, with $5 \%$ level of significance and $95 \%$ confidence interval.

\section{RESULTS}

60 patients with palpable breast lumps underwent sonomammogram, out of which 33 cases (55\%) were reported as benign and 19 cases (31.7\%) were reported as malignant and 8 cases were reported as indeterminate (can be either benign or malignant) by ultrasound. As indeterminate results are neither false positive or false negative, it recommends the further need of biopsy to rule out malignancy These indeterminate cases were not included into calculation. 52 palpable lumps were included in the study. The specificity, sensitivity, negative predictive value, positive predictive value of ultrasound is $94.3 \%, 100 \%, 100 \%, 89.5 \%$ respectively. False negative rate and false positive rate of USG is $0 \%$ and $5.7 \%$ respectively (Table 1 ).

Fine Needle Aspiration Cytology was done for all 60 palpable masses and 42 cases were reported as benign (70\%), and 17 cases (28.3\%) were reported as malignant and in 1 case $(1.7 \%)$ the result was indeterminate. As indeterminate result is neither false positive or false negative, it recommends the further need of biopsy to rule out malignancy These indeterminate cases were not included into calculation. The sensitivity of FNAC was calculated for 59 lumps. The specificity, sensitivity, negative predictive value, positive predictive value of FNAC is $100 \%, 85 \%, 92.9 \%, 100 \%$ respectively. False negative and false positive rate of FNAC is $7.1 \%$ and $0 \%$ respectively (Table 1 ). Table 2 shows final histopathological diagnosis of benign masses and malignant breast masses. The most commonly diagnosed benign breast mass is fibroadenoma and the most common malignancy was infiltrating ductal carcinoma.

The ultrasound features which were more predictive for a benign lesion (Figure 1) were oval, < 3 smooth macro lobulations $(94.4 \%$ of masses with these features were diagnosed as benign), circumscribed margins ( $95 \%$ of masses with feature were benign), Width - to - AP ratio >1.4 (81.6\% of masses with W/ A-P ratio > 1.4 were benign), pseudo capsule ( $96.2 \%$ of lesions with pseudo capsule were benign), edge refraction $(82.4 \%$ lesions with edge refraction were benign). $100 \%$ of the lesions with round shape were benign but round shape was reported only in $5 \%$ of cases.

The features which were more predictive for a malignant lesion (Figure 2) were irregular shape ( $85.7 \%$ of lesions with this feature were malignant), non-circumscribed (spiculated, angular, indistinct, micro lobulated) margins (90\% of lesions with non-circumscribed margins were malignant), Width to - AP ratio < 1.4 (100\% of lesions with Width - to - AP ratio $<1.4$ were malignant).

The USG imaging parameter which is not a good reliable factor in differentiation of masses is echo-pattern as in our study $53.8 \%$ of masses with hypoechoic echo pattern were benign and $46.2 \%$ were malignant. Certain features showed very good correlation in categorization of benign and malignant lesions were too in frequent to be applicable generally. For example, a hyperechoic lesion is a very good predictor of a benign mass but it is seen in only $3 \%$ of the cases.

\begin{tabular}{|l|c|c|}
\hline Parameters & USG & FNAC \\
\hline Sensitivity & $100 \%$ & $100 \%$ \\
\hline Specificity & $94.3 \%$ & $100 \%$ \\
\hline Positive Predictive Value (PPV) & $89.5 \%$ & $92.9 \%$ \\
\hline Negative Predictive Value (NPV) & $100 \%$ & $0 \%$ \\
\hline False Positive (FP) & $5.7 \%$ & $7.1 \%$ \\
\hline False Negative (FN) & $0 \%$ & \\
\hline \multicolumn{2}{|c|}{ Table-1: Specificity, sensitivity, negative predictive value, positive predictive value, false negative and false positive results of ultra- } \\
\end{tabular}

\begin{tabular}{|c|c|c|c|}
\hline Histopathology & Diagnosis & Frequency & Percentage \\
\hline \multirow[t]{7}{*}{ Benign } & Breast abscess & 2 & 3.3 \\
\hline & Fibroadenoma & 30 & 50.0 \\
\hline & Galactocele & 1 & 1.7 \\
\hline & Hamartoma & 2 & 3.3 \\
\hline & Hematoma & 1 & 1.7 \\
\hline & Phyllodes & 3 & 5 \\
\hline & Lipoma & 1 & 1.7 \\
\hline \multirow[t]{6}{*}{ Malignant } & Invasive papillary carcinoma & 1 & 1.7 \\
\hline & Lobular carcinoma & 3 & 5.0 \\
\hline & Medulary ca & 1 & 1.7 \\
\hline & Infiltrating ductal carcinoma & 14 & 23 \\
\hline & Malignant phyllodes & 1 & 1.7 \\
\hline & Total & 60 & 100.0 \\
\hline
\end{tabular}



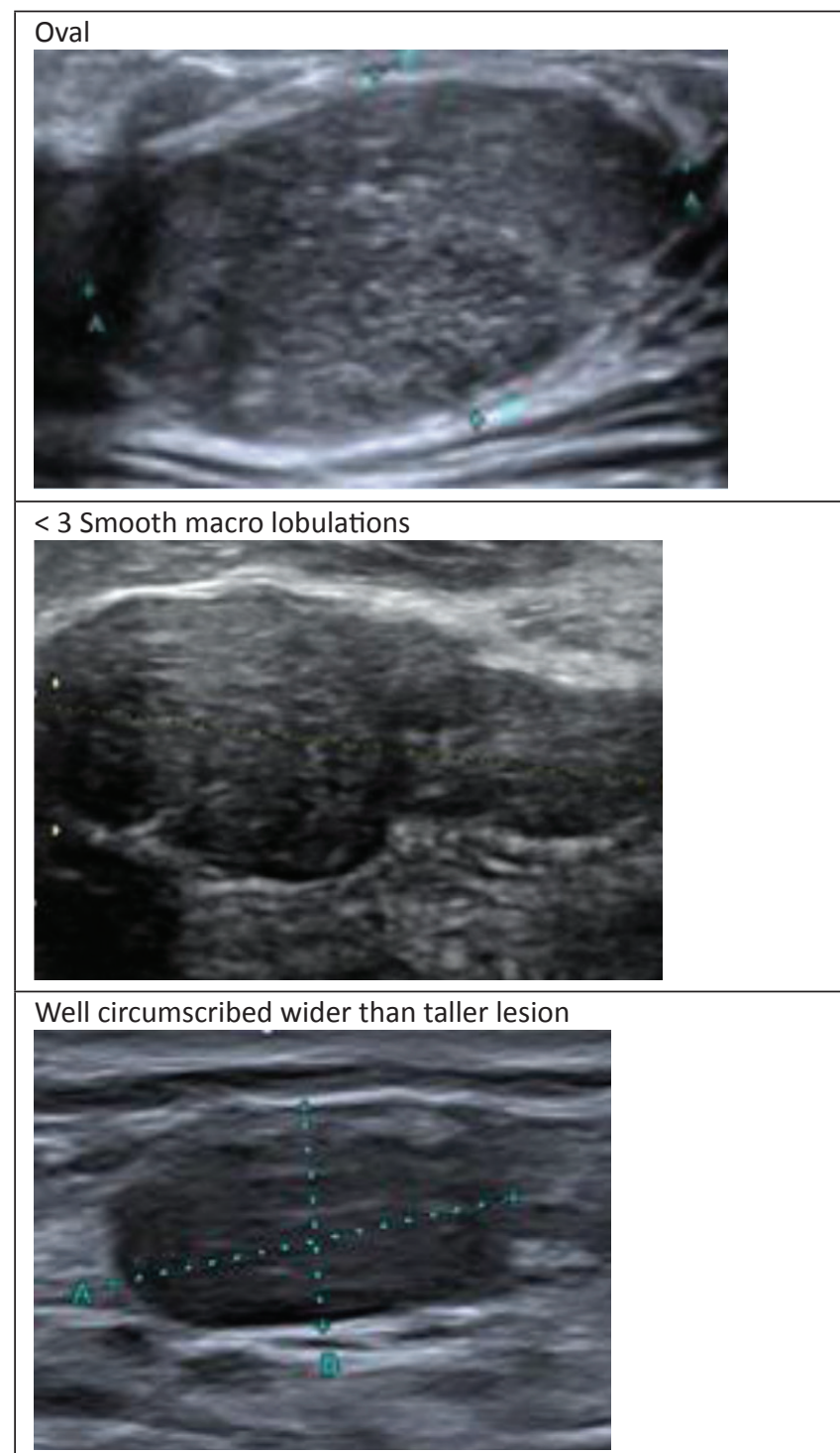

Presence of posterior acoustic enhancement

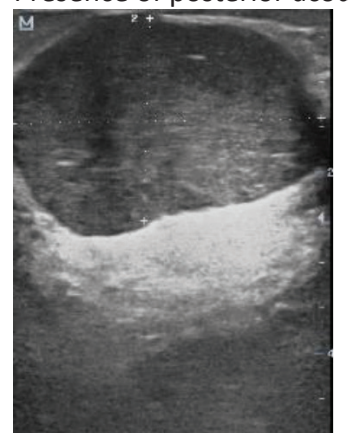

Presence of edge refraction

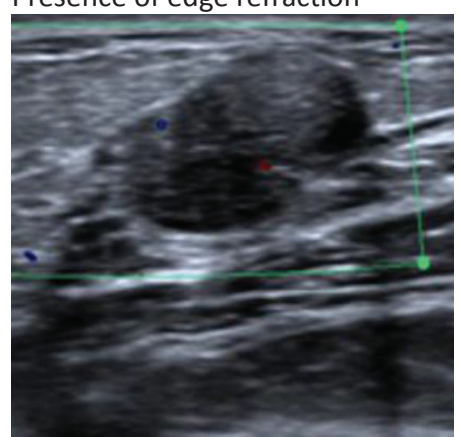

Figure-1: Characteristics of a benign lesion
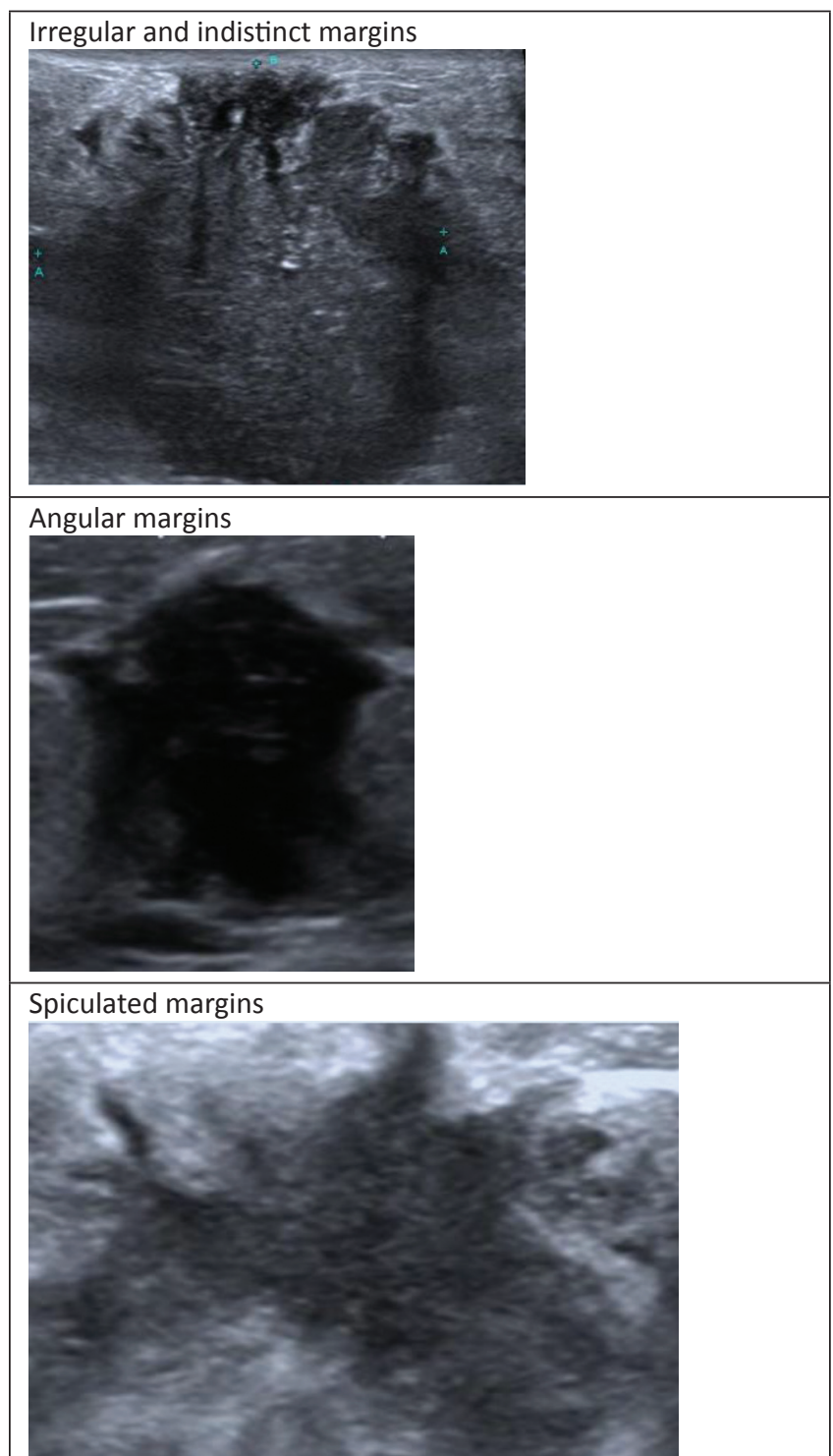

Taller than wider lesion

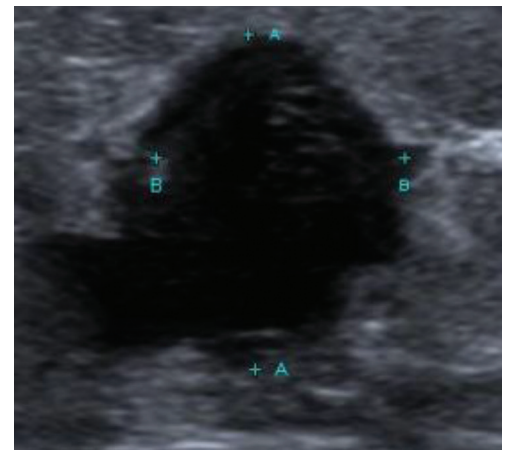

Figure-2: Characteristics of a malignant lesion

\section{DISCUSSION}

In our study the sensitivity of sono-mammogram in the detection of malignant breast masses is found to be $100 \%$ for the 52 breast lumps excluding 8 indeterminate cases. The sensitivity of our study shows good correlation with the study conducted by Stavros et $\mathrm{a}^{12}$, were the sensitivity was found to be $98.4 \%$. The sensitivity of USG in the detection of malignant breast masses ranges between 67 to $98.4 \%$ in previous studies. ${ }^{15,16,17}$ This wide range of difference 
in sensitivity is due to various reasons, such as different sample size, case selection criteria, ultrasound machine parameters like different resolution power and the skill of the Radiologist. Negative predictive value of ultrasonogram is $100 \%$. This shows good correlation with a study by Soo \& Rosen et $a 1^{18}$ in which the NPV was found to be $99.8 \%$. The sensitivity of ultrasound in diagnosing malignancy for 52 palpable breast masses included in our study is $100 \%$, this means that ultrasound identified all malignant lesions as malignant when compared with tissue diagnosis results. The specificity of ultrasound is $94.3 \%$. This shows that ultrasound diagnosed 2 benign lesions as malignant which was confirmed by tissue diagnosis. The positive predictive value was $89.5 \%$. In the present study ultrasound was able to categorize $86.6 \%$ of the total masses as either malignant or benign with a false positive rate of $(5.7 \%)$ and false negative rate of $(0 \%)$. This shows that ultrasound is good imaging modality for diagnosing malignant palpable breast masses. Ultrasound was giving indeterminate results in $13 \%$ cases.

In the present study the calculated sensitivity of FNAC in the diagnosis of malignant breast lesions for 59 cases is found to be $85 \%$. FNAC result was indeterminate in one of the breast masses, and so that case was excluded from the calculation as indeterminate results are neither false positive or false negative. The sensitivity of FNAC in the diagnosis of malignant breast masses in previous studies ranges between $68 \%$ to $97.4 \% .^{15,16,17}$ Our result correlates well with previous studies. The wide variations in sensitivity of FNAC in previous studies is due to different inclusion parameters. The other factors that would be site of the lesion, size of the lesion, whether palpable or non-palpable, the varying echogenicity of the lesion and the experience of the pathologist interpreting the FNAC. The specificity of FNAC in present study is $100 \%$, that shows that FNAC diagnosed all benign lesions as benign. Positive predictive value is found to be $(100 \%)$, and negative predictive value was found to be $(92.9 \%)$. The false positive rate is $0 \%$, false negative rate is 7.1\%. This correlated well with previous studies.

When we compare Ultrasound and FNAC findings, the sensitivity of ultrasound was found to be (100\%), the sensitivity of FNAC was found to be (85\%), the false negative rate of sono-mammogram was found to be (5.7\%), the false negative rate of FNAC was found to be (7.1\%). These results signify that ultrasound is a better modality for diagnosing malignant lesions. But the percentage of indeterminate results in ultrasound is high (13\%) when compared to FNAC (1.6\%). Percentage of indeterminate results were very less by FNAC. Out of the 20 malignant breast masses, FNAC was able to diagnose malignancy correctly in 17 cases (85\%), in the rest of the 3 cases (15\%) of malignancy was reported as benign by FNAC. The results of ultrasound in these 3 cases were compared with FNAC. In all 3 cases ultrasound has diagnosed malignancy correctly. And out of 60 cases FNAC showed indeterminate result in 1 case which turned to be phyllodes tumour in biopsy. The false negative rate of FNAC showed good correlation with study conducted by Day et $\mathrm{al}^{18}$ and Feichter et $\mathrm{al}^{19}$

USG was not able to accurately categorize benign and malignant lesions in $13 \%$ of the cases. In all these cases, FNAC was able to exactly identify benign lesions and malignant lesions which was further confirmed by histopathology. This states that in case of indeterminate reports by ultrasound further FNAC is needed to rule out or confirm malignancy. Thus, when both ultrasound and FNAC are used together, majority of the lesions can be diagnosed. In our study the agreement between ultrasound and FNAC is found to be $82.6 \%$. Ultrasound was able to diagnose all the malignant cases missed by FNAC and in case of indeterminate reports by ultrasound, FNAC could diagnose benign and malignant lesions correctly. Thus, both these modalities are considered complementary to each other. When USG and FNAC are applied together the need of surgical biopsy for benign lesions can be reduced. Another similar study was conducted by Reinikainen et $\mathrm{al}^{20}$, to evaluate the application of USG and FNAC in the assessment of palpable solid breast lesions. USG and mammography images of (84 palpable breast lesions) and 57 fine needle aspiration cytology results were analysed retrospectively. Comparison of both the modalities were done with histopathological diagnosis. The specificity and sensitivity of FNAC were $83 \%$ and $92 \%$ respectively. They concluded that correct applications of these three modalities can reduce the number of biopsies of benign lesions. ${ }^{21}$

Stavros et al conducted a study with 750 female patients with breast masses, NPV (Negative predictive value), sensitivity of FNAC of the study conducted by stavros et al was $98.4 \%$ and 99.5\% respectively. This showed good correlation with the present study. The percentage of benign and malignant breast masses in study conducted by Stavros et al was $67 \%$ and $33 \%$ respectively. Majority of the lesions were benign which well correlated with the study done by Stavros et al. ${ }^{12}$ The most commonly diagnosed benign lesion is fibroadenoma and the most commonly encountered malignancy is infiltrating ductal carcinoma.

The ultrasound features which were more predictive for a benign lesion in our study were oval, < 3 smooth macro lobulations ( $94.4 \%$ of lesions with this feature were diagnosed as benign), circumscribed margins (95\% of lesions with feature were benign), Width- to- AP ratio $>1.4$ (81.6\% of lesions with this feature were benign), pseudo capsule $(96.2 \%$ of lesions with pseudo capsule were benign), edge refraction (82.4\% lesions with edge refraction were benign). 100\% of the lesions with round shape were benign but round shape was reported only in $5 \%$ of cases.

The features which were more predictive for a malignant lesion were irregular shape $(85.7 \%$ of lesions with this feature were malignant), non-circumscribed (spiculated, angular, indistinct, micro lobulated) margins (90\% of lesions with non-circumscribed margins were malignant), Width to - AP ratio $<1.4 /$ taller than wider $(100 \%$ of lesions with Width - to -AP ratio < 1.4 were malignant). These features show very good correlation with a study conducted by Rahbar et al in 1999.22

Rahbar et al conducted a study to categorize benign and malignant lesions by ultrasound. In this study they concluded certain features such as round or oval shape, well circumscribed margins, ratio of width to antero-posterior dimension more than 1.4 as the most reliable features of identification of benign lesions. And the features that characterize malignant 
masses were irregular shape, spiculated or micro lobulated margins ratio of width to Antero - posterior dimension $<1.4$. The overall cancer biopsy yield has increased when 3 most reliable criteria has been applied by each Radiologist strictly. They concluded that some ultrasound features would help in accurate identification of benign and malignant masses. The interpreter variability should be observed before applying these criteria generally to defer biopsy of solid masses.

In our study $67 \%$ of lesions had circumscribed margins and $33 \%$ of lesions had non-circumscribed margins. When the lesions with circumscribed margins were analysed 95\% of the lesions turned to be benign and $5 \%$ of lesions turned to be malignant. This shows that circumscribed margins are an important predictor of benign lesions. Out of the $33 \%$ of lesions which showed non-circumscribed margins, $90 \%$ were malignant and $10 \%$ were benign. This shows that non circumscribed margins (Angular, spiculated, indistinct, micro lobulated) is an important predictor of malignancy. 2 cases (10\%) each one with angular and spiculated margins respectively turned to be benign and the final diagnosis was chronic breast abscess. This shows that breast abscess can mimic malignancy. Chronic idiopathic granulomatous mastitis may manifest initially in the breast and mimic an infectious process or malignancy. ${ }^{23}$

During 1996, Al-Dabbagh AA, Al-Baghdadi TM ${ }^{24}$ conducted a study for evaluating malignant breast masses. 114 patients were examined and out of which 107 patients had features suspicious for malignancy. The wall contour, echo pattern, echogenicity, posterior shadowing, width to length ratio were the parameters taken for assessment. With these features the positive detection rate for malignancy was $91.2 \%$. They concluded that ultrasound can be used in assessment of breast masses.

In our study we identified the 3 best reliable features for identification of benign and malignant lesions. These features were the margins, shape, ratio of width to AP dimension of the breast lump. These features show very good correlation with the previous studies. Stavros et al emphasized the importance of criteria which should be strictly applied for identification of malignant and benign lesions.

We believe that our study has several strengths. First, such types of studies are done in a limited basis on Indian populations. So, our study will be very useful in reference to the Indian oriented studies. Second, our study strengthens the importance of sono- mammography in evaluation of breast masses which proves the results of previous studies results. Thirdly, the results were compared with gold standard histopathological diagnosis. For a valid study always the reference test which is evaluated must me gold standard. And the strong point in our study would be the Radiologist performing the Ultrasound guided FNAC and the cytopathologist interpreting the FNAC were blinded from each other's observations. And both the diagnostic procedure was done in the same population this adds further strength to the study. First limitation of our study would be is the single observer interpretation. So, we did not assess interobserver difference in the assessment of these features and in the final assessments. Secondly, we excluded cystic lesions in our study as they were managed by aspiration and drainage and tissue diagnosis is not possible. This selectiveness of patients in the study may limit the generalisation of the findings in clinical practice.

\section{CONCLUSION}

Ultrasonography helps in early detection of breast malignancy in young and pregnant women. Importance of breast imaging is not to miss malignant lesion in early stage. In our study the agreement between ultrasound and FNAC is found to be $82.6 \%$. Ultrasound was able to diagnose all the malignant cases missed by FNAC and in case of indeterminate reports by ultrasound FNAC was able to diagnose benign and malignant lesions accurately. Thus, both the modalities are considered complementary to each other. When Ultrasound and FNAC are used together in the diagnosis of benign and malignant breast lesions the need of surgical biopsy for benign lesions can be reduced. Factors which were more predictive of benign and malignant features were identified with ultrasound. These features have the potential to help decrease the number of biopsies performed for benign solid masses. Our study results were encouraging in that we were able to identify the important ultrasound features for differentiating malignant and benign masses. These features have the potential to help us to decrease the need for radiation exposure on mammography $\&$ the number of biopsies performed for benign solid masses. Thus, ultrasound is a useful supplement to FNAC/ Biopsy in diagnosis of benign and malignant lesions.

\section{REFERENCES}

1. World Health Organization. Global action plan for the prevention and control of noncommunicable diseases 2013-2020.

2. Anderson BO, Yip CH, Smith RA, Shyyan R, Sener SF, Eniu A, Carlson RW, Azavedo E, Harford J. Guideline implementation for breast healthcare in low-income and middle-income countries. Cancer. 2008;113(S8):222143.

3. Clopton MB. The difficulty of diagnosis of carcinoma of the breast. Radiology 1925;4(5):390-394.

4. Cole-Beuglet C, Beique RA. Continuous ultrasound B-scanning of palpable breast masses. Radiology 1975;117(1):123-128.

5. Damascelli B,Musumeci R, Orefice S. Sonar information about breast tumors. Radiology 1970;96(3):583-586.

6. Kobayashi T. Gray-scale echography for breast cancer. Radiology 1977;122(1):207-214.

7. Meritt CRB. Technology Update. Radiol Clin North Am. 2001;39(3):385-97.

8. Shapiro RS, Wagreich J, Parsons RB, et al. Tissue Harmonic Imaging Sonography, evaluation of image quality compared with conventional sonography. AJR. 1998;171(4):1203-6.

9. Zhi H, Ou B, Luo BM, Feng X, Wen YL, Yang HY. Comparison of Ultrasound Elastography, Mammography, and Sonography in the Diagnosis of Solid Breast Lesions. J Ultrasound Med. 2007;26(6):807-15.

10. Crystal P, Strano SD, Shcharynski S, Koretz MJ. Using Sonography to Screen Women with Mammographically 
Dense Breasts. AJR Am J Roentgenol.2003;181(1):17782.

11. Spak DA, Plaxco JS, Santiago L, Dryden MJ, Dogan BE. BI-RADS ${ }^{\circledR}$ fifth edition: A summary of changes. Diagnostic and interventional imaging. 2017;98(3):17990.

12. Jackson VP. Management of solid breast nodules: what is the role of sonography?. Radiology. 1995;196(1):14-5.

13. Chen DR, Chang RF, Huang YL. Breast cancer diagnosis using self-organizing map for sonography. Ultrasound in medicine \& biology. 2000;26(3):405-11.

14. Rao AA, Feneis J, Lalonde C, Ojeda-Fournier H. A pictorial review of changes in the BI-RADS fifth edition. Radiographics. 2016;36(3):623-39.

15. Jayaram G, Alhady SF, Yip CH. Cytological analysis of breast lesions: A review of 780 cases. Malaysian Journal of Pathology. 1996;18(2):81-87.

16. Soo MS, Baker JA, Rosen EL. Sonographic detection and sonographically guided biopsy of breast microcalcifications. American Journal of Roentgenology. 2003;180(4):941-8.

17. Mendoza P, Lacambra M, Tan PH, Tse GM. Fine needle aspiration cytology of the breast: the nonmalignant categories. Pathology Research International. 2011;2011.

18. Day C, Moatamed N, Fimbres AM, Salami N, Lim S, Apple SK. A retrospective study of the diagnostic accuracy of fine-needle aspiration for breast lesions and implications for future use. Diagnostic Cytopathology. 2008;36(12):855-860.

19. Feichter GE, Haberthür F, Gobat S, Dalquen $P$. Breast cytology: statistical analysis and cytohistologic correlations. Acta Cytologica. 1997;41(2):327-332.

20. Reinikainen HT, Rissanen TJ, Piippo UK, Päivänsalo MJ. Contribution of ultrasonography and fineneedle aspiration cytology to the differential diagnosis of palpable solid breast lesions. Acta Radiol. 1999;40(4):383-89

21. Apple SK, Dascalos JM, Bassett LW. Infectious and inflammatory diseases of the breast. In: Bassett LW, Mahoney MC, Apple SK, D’Orsi CJ, eds. Breast imaging. Expert Radiology Series. Philadelphia, Pa: Saunders Elsevier, 2011; 375-390.)

22. Rahbar G, Sie AC, Hansen GC, Prince JS, Melany ML, Reynolds HE, Jackson VP, Sayre JW, Bassett LW. Benign versus malignant solid breast masses: US differentiation. Radiology. 1999;213(3):889-94.

23. Sabaté JM, Clotet M, Gómez A, De Las Heras P, Torrubia S, Salinas T. Radiologic evaluation of uncommon inflammatory and reactive breast disorders. RadioGraphics 2005;25(2):411-424.

24. Al-Dabbagh AA, Al-Baghdadi TM. Evaluation of the sonographic features of malignant breast masses. Annals of Saudi medicine. 1996;16(2):119-22.

Source of Support: Nil; Conflict of Interest: None

Submitted: 18-02-2020; Accepted: 13-04-2020; Published online: 10-05-2020 